\title{
Gene delivery and gene expression in vertebrate using baculovirus Bombyx mori nucleopolyhedrovirus vector
}

\author{
Xingjian Liu' ${ }^{1, *}$, Yinü $\mathrm{Li}^{1{ }^{1, *}}$, Xiaoyuan $\mathrm{Hu}^{1}$, Yongzhu $\mathrm{Yi}^{2}$ and Zhifang Zhang ${ }^{1}$ \\ ${ }^{1}$ Biotechnology Research Institute, Chinese Academy of Agricultural Sciences, Beijing, China \\ ${ }^{2}$ The Sericultural Research Institute, Chinese Academy of Agricultural Sciences, Zhenjiang, China \\ *These authors have contributed equally to this work \\ Correspondence to: Zhifang Zhang, email: zhifangzhang@yahoo.com \\ Keywords: Bombyx mori nucleopolyhedrovirus; complement resistance; gene delivery \\ Received: July 06, $2017 \quad$ Accepted: October 28, $2017 \quad$ Published: November 20, 2017 \\ Copyright: Liv et al. This is an open-access article distributed under the terms of the Creative Commons Attribution License 3.0 (CC \\ BY 3.0), which permits unrestricted use, distribution, and reproduction in any medium, provided the original author and source are \\ credited.
}

\section{ABSTRACT}

The baculovirus Autographa californica multicapsid nucleopolyhedrovirus (ACMNPV) has been investigated as a possible tool for gene therapy, but its inhibition by complement proteins in human serum limits its applicability. Here, we used the baculovirus Bombyx mori nucleopolyhedrovirus (BmNPV) to construct a gene delivery vector in which a reporter gene is driven by a cytomegalovirus IE promoter. Enhanced green fluorescent protein (EGFP) and luciferase reporter genes were used to test the efficiency of gene delivery. In vitro complement inactivation data showed that the recombinant $B m N P V$ vector was more stable in human serum than the recombinant $A C M N P V$ vector. The recombinant $B m N P V$ vector successfully delivered the reporter genes into different tissues and organs in mice and chicks. These results demonstrate that the BmNPV vector is more stability against complement inactivation in human serum than the AcMNPV vector, and indicate that it may be useful as an effective gene delivery vector for gene therapy in vertebrates.

\section{INTRODUCTION}

The idea of gene therapy was originally conceived in the 1970s [1,2]. The feasibility was later confirmed by experiments in mice [3], and the first clinical application in humans was performed in the 1990s [4]. The method continues to show promise for treating inherited diseases and cancer [5-7].

The gene delivery system used to deliver nucleic acids into target cells is a key component in determining the clinical success of a gene therapy treatment. The most efficient delivery systems are based on viruses that infect animal cells, such as lentiviruses, retroviruses, adenoviruses and adeno-associated viruses [8]. Baculoviruses have been also explored as a possible gene delivery vector system for vertebrate cells [9-12]. These viruses may be ideal for gene therapy because their viral promoters are almost silent in mammalian cells, and the budded viral form is harmless to the environment [13-16]. Autographa californica multicapsid nucleopolyhedrovirus (AcMNPV), a widely studied baculovirus, is as a promising gene therapy vector
[17]. Previous in vivo studies have demonstrated that $A c \mathrm{MNPV}$ directly injected into target tissues, including brain and testis, can drive the foreign gene expression in rats and mice [18-21]. A single intravitreal injection of recombinant baculovirus could induce target gene expression in eye [22,23]. In addition, the $A c$ MNPV vector has been investigated as a possible tool for cancer gene therapy [24-26]. However, AcMNPV is inhibited by the complement proteins in human serum, which restricts its applications for gene delivery in vivo [27].

Bombyx mori nucleopolyhedrovirus (Bm NPV) is another baculovirus with a limited host range $[28,29]$. This virus also drives the expression of target genes in mammalian cells [30]. In this study, we used BmNPVderived recombinant baculoviruses to deliver reporter genes into mice and chicks. The cytomegalovirus (CMV) IE promoter [31] was chosen to drive reporter gene expression in vivo. Our in vitro and in vivo results demonstrate that the $B m \mathrm{NPV}$ vector exhibits a greater stability against complement inactivation in human serum than the $A c \mathrm{MNPV}$ vector. 


\section{RESULTS}

\section{Construction of reporter genes delivery vectors}

We constructed Baculovirus transfer vectors containing CMV IE promoter based on the pVL1393 vector. The polyhedron promoter of the pVL1393 transfer vector was replaced with the CMV IE promoter. SV40 polyadenylation signal was inserted downstream the multiple cloning site. The CMV promoter transfer vector was named pVLCMV (Figure 1A). Luciferase and enhanced green fluorescent protein (EGFP) genes were separately inserted into the vector (Figure 1B). The recombinant baculoviruses reBm-luc and reBm-EGFP, used to deliver the luciferase and EGFP genes, respectively, were prepared by using cotransfection of reBmBac and pVLCMV-luc or pVLCMV-EGFP in Bm cells. The recombinant baculovirus vector reAc-luc was used for comparison assays of complement inactivation. The vector was prepared by cotransfection of BacPAK6 and pVLCMV-luc.

Fifth instar silkworm larvae and Sf9 cells were infected with the recombinant baculoviruses for amplification. The recombinant baculoviruses vectors were amplified in larval hemolymph and cell-culture supernatant,
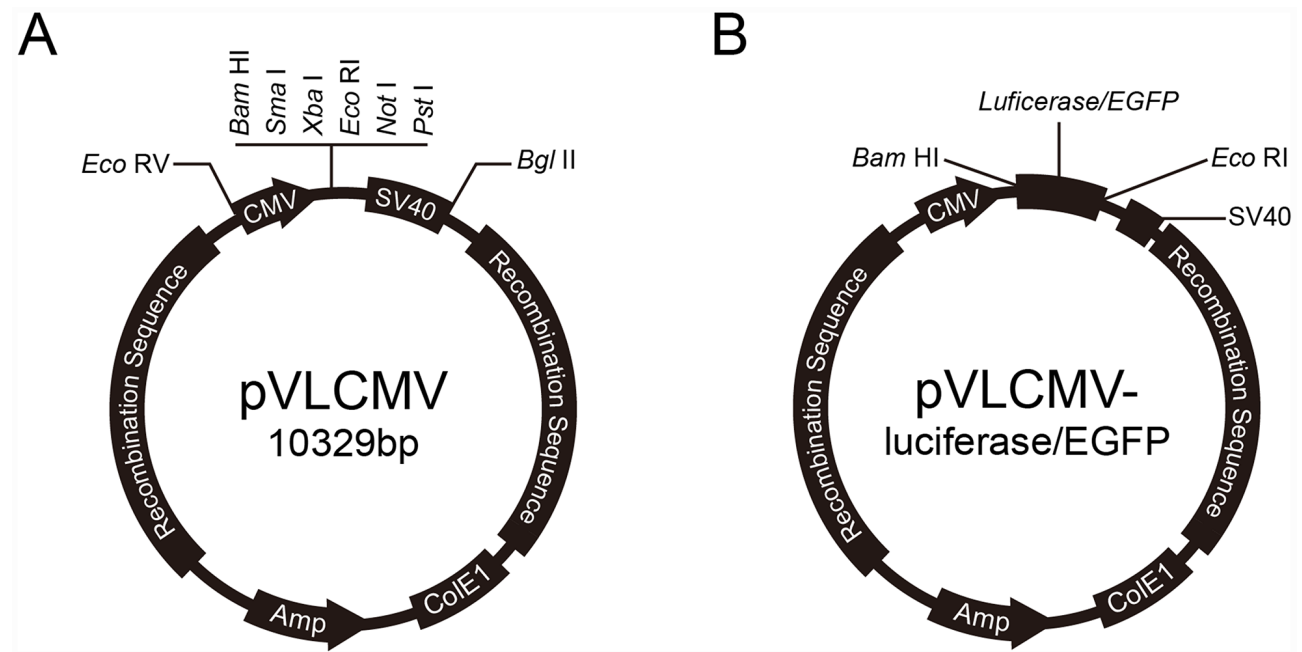

Figure 1: Reporter genes transfer vector.

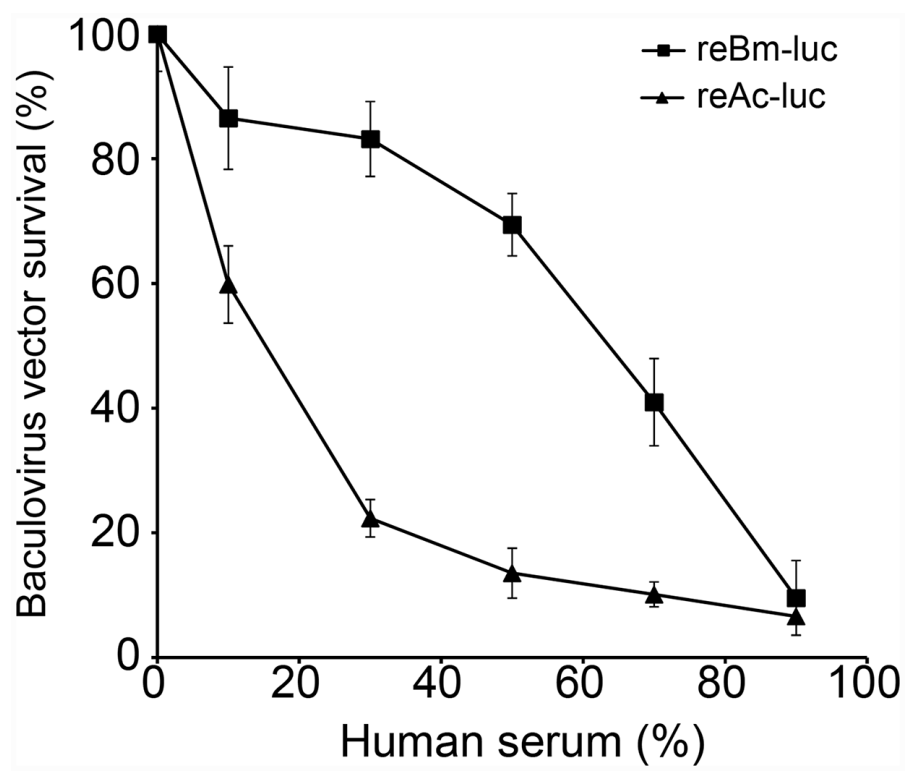

Figure 2: Baculovirus survival in human serum. Recombinant $B m \mathrm{NPV}$ showed stronger resistance to complement inactivation in human serum than recombinant $A c \mathrm{MNPV}$. 
purified, and concentrated by ultracentrifugation. Purified viruses were quantified by real-time PCR (Q-PCR) to determine the amount of viral genome per milliliter (vg/ $\mathrm{mL}$ ). The quantified results for the reporter genes were consistent with those of the viral genome, which made it possible to determine the purity of the recombinant viruses and number of copies of the viral genome.

\section{Resistance to complement inactivation}

The reBm-luc recombinant baculovirus was used to measure resistance to complement inactivation in human serum. Compared with luciferase gene delivery $A c \mathrm{MNPV}$ (reAc-luc), reBm-luc exhibited increased stability in human serum (Figure 2). When the serum concentration reached $50 \%$, the survival rate of reBm-luc was approximately $70 \%$, whereas reAc-luc exhibited $16 \%$ survival. These results somewhat differ from a previous report by Hofmann et al [26]; this difference might have been caused by technical differences and different cells used. The results for $A c \mathrm{MNPV}$ were consistent.

\section{EGFP gene expression in vitro and in vivo}

VERO, PK-15, HEK293T, and Hela cells can be transduced by recombinant baculovirus. Here, we used these cell lines to verify the gene delivery in vitro. Fluorescence imaging of reBm-EGFP transduced cells shows the EGFP expression (Supplementary Figure 1).

The recombinant baculovirus reBm-EGFP was used to transfect mice and chicks. Fluorescence imaging of mouse tissue slices indicated that EGFP was effectively expressed in the lungs, spleens, kidneys, and brains (Figure 3 ) from the $5^{\text {th }}$ day to the $17^{\text {th }}$ day after intravenous injection (IV) or intragastric administration (IG). Immunoblotting analysis verified the protein expression of EGFP-constructs in these organs (Figure 5A). Similar results were observed in the lungs, spleens,
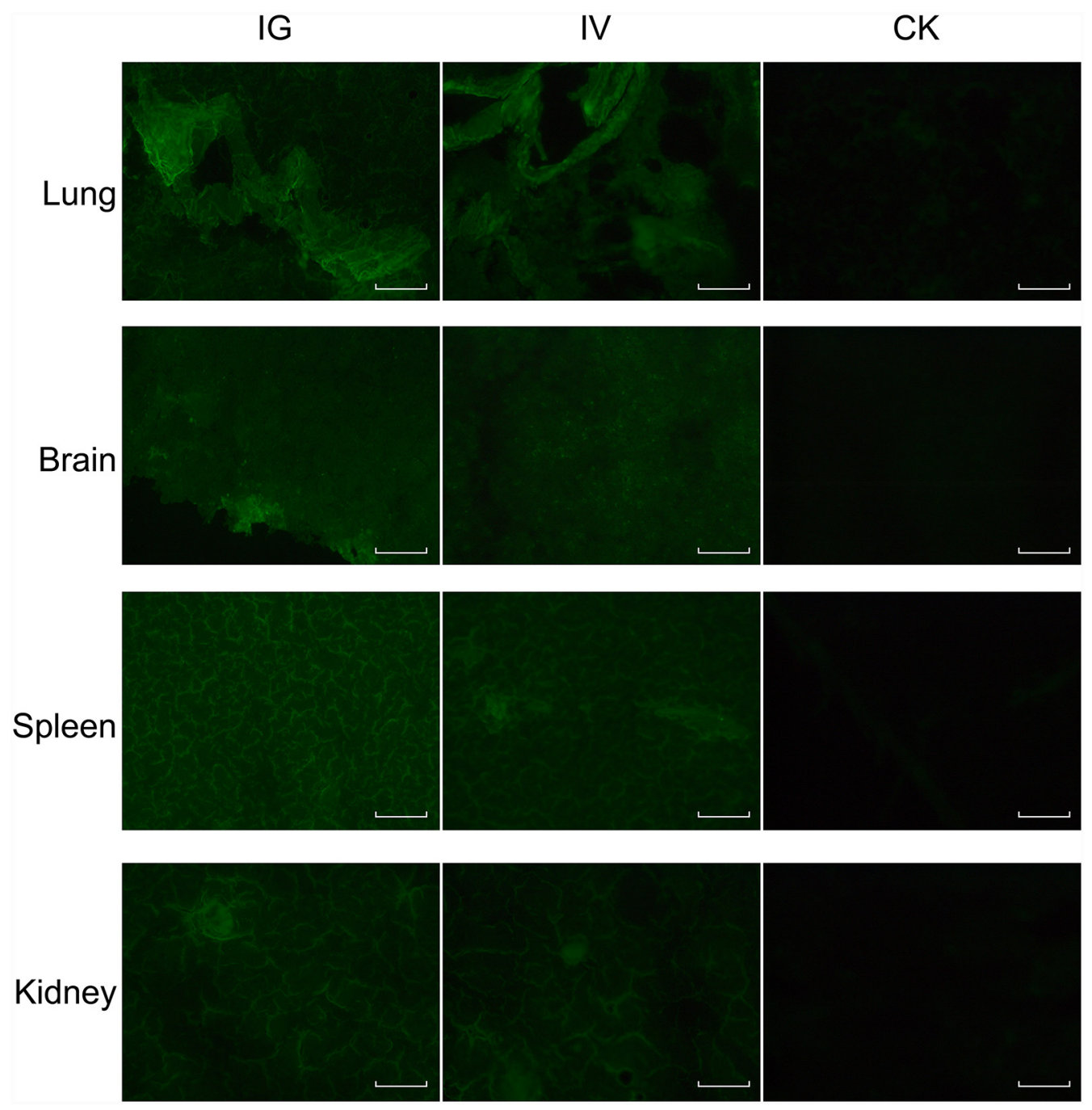

Figure 3: EGFP expression in mice. Abbreviations: IG, intragastric administration group; IV, intravenous injection group; CK, control group. Scale bar, $100 \mu \mathrm{m}$. 
kidneys, bursa of Fabricius, and brains of chicks (Figure 4 and Figure 5B). After the 21st day, no EGFP expression was observed in the above tissues in mice and chicks. However, in the intramuscular injection (IM) groups, EGFP was not detected. In addition, the fluorescence background in liver was too strong to identify any specific EGFP expression. Due to the presence of fluorescence in most tissues, a luciferase reporter gene delivery assay was performed to verify the EGFP results.

\section{Luciferase gene expression in vivo}

Mice and chicks tissues were collected and assayed for luciferase activity at 5, 11, 17, 21, and 30 days after reBm-luc intravenous (IV), intramuscular (IM), or
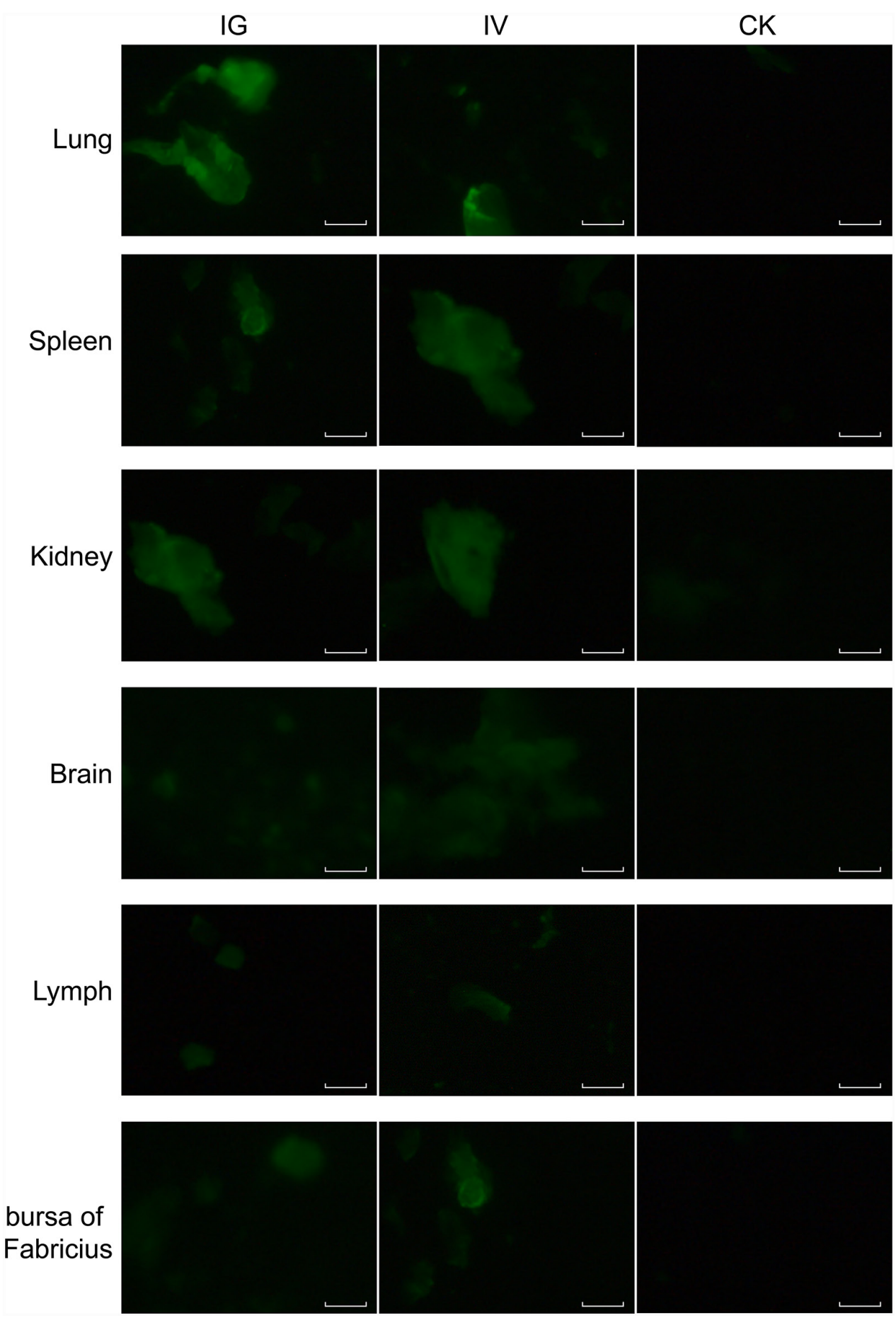

Figure 4: EGFP expression in chicks. Abbreviations: IG, intragastric administration group; IV, intravenous injection group; CK, control group. Scale bar, $100 \mu \mathrm{m}$. 
intragastric (IG) administration. In the mouse IV and IG groups, luciferase activity was observed in lung, liver, kidney, spleen, and brain at 5 and 11 days (Figure 6A). No significant luciferase activity was detected in these tissues after 17 days. In chicks of the IV and IG groups, luciferase activity was detected in lung, liver, kidney, bursa of Fabricius, pancreas, lymphonodus, and brain at 5 and 11 days (Figure 6B). After 17 days, the luciferase activity disappeared. No luciferase activity was detected in mouse muscle tissues. However, a weak activity was detected in chicks of the IG and IV groups. No luciferase expression was detected in mice or chicks IM groups.

\section{DISCUSSION}

Our results indicate that the $B m \mathrm{NPV}$ vector is more stable and resistant to inactivation by the human serum complement compared with the AcMNPV vector. The $A c \mathrm{MNPV}$ baculovirus vector has been widely used for gene delivery, but it is complement-sensitive [26]. Thus, in the gene therapy field, baculoviruses have been used only to deliver target genes into tissues in which the viruses are not exposed to complement proteins [19, 20, 22, 32], and to prepare recombinant adeno-associated virus (rAAV)containing target genes $[33,34]$. Numerous gene therapy

A

\begin{tabular}{|c|c|c|c|c|c|c|c|c|c|c|c|c|}
\hline & \multicolumn{3}{|c|}{ Lung } & \multicolumn{3}{|c|}{ Brain } & \multicolumn{3}{|c|}{ Spleen } & \multicolumn{3}{|c|}{ Kidney } \\
\hline & IG & IV & CK & IG & IV & CK & IG & IV & CK & IG & IV & CK \\
\hline EGFP & $=$ & - & & - & - & & $\cdots$ & $=$ & & - & - & \\
\hline
\end{tabular}

B

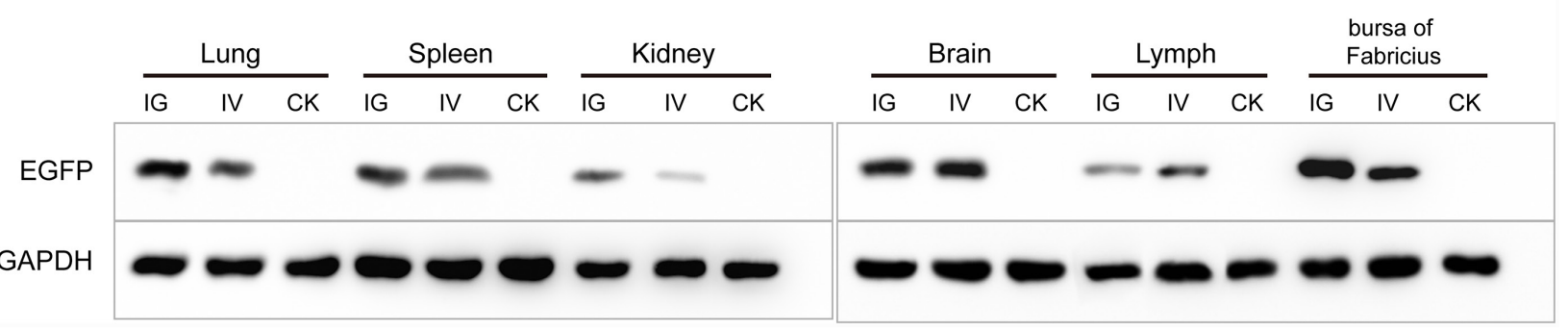

Figure 5: The Western blotting analysis of EGFP in mice (A) and chicks (B). Abbreviations: IG, intragastric administration group; IV, intravenous injection group; CK, control group.

A

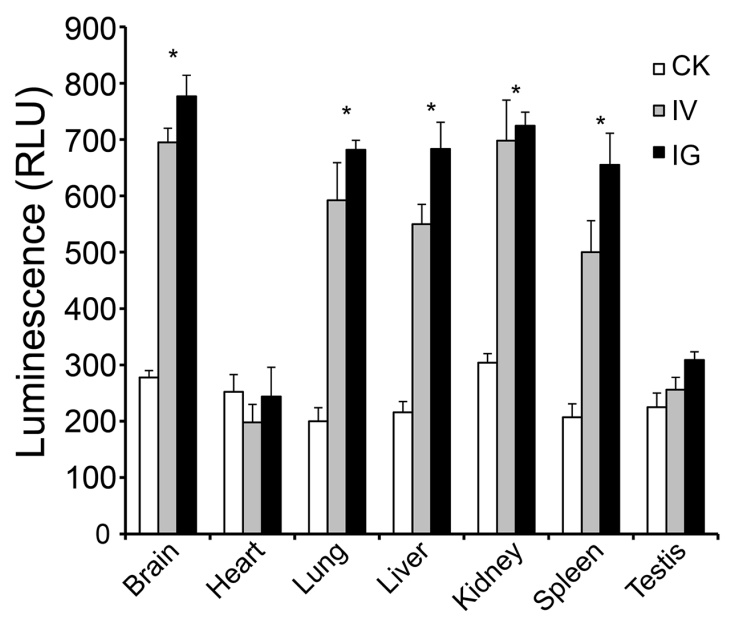

B

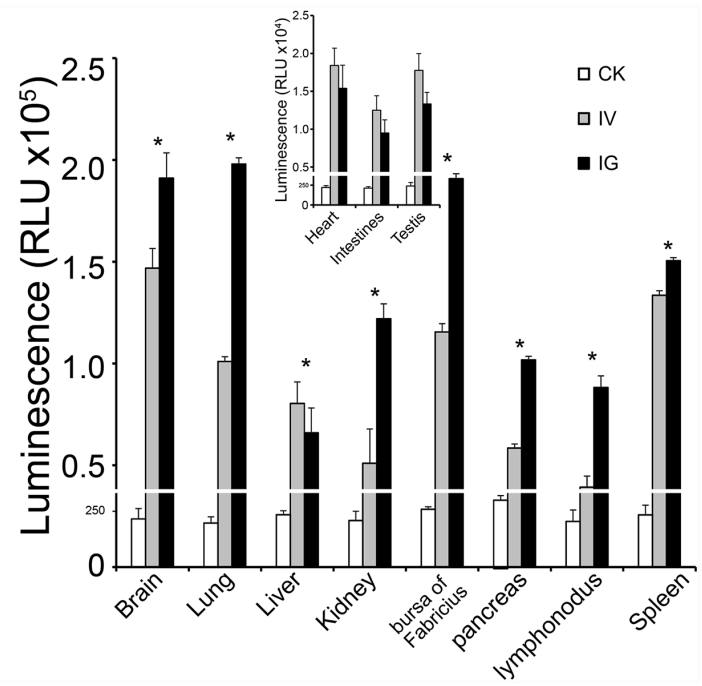

Figure 6: Luciferase activity in vivo. (A) Luciferase activity was detected in mouse brain, lung, liver, kidney and spleen. (B) Luciferase activity was detected in most tissues in chicks. The expression efficiency in chicks was higher than in mice. 
trial in vivo studies have used the $A c \mathrm{MNPV}$ vector, even in cancer gene therapy [24-26], but its complement sensitivity restricts its further clinical use. Many strategies have been devised to overcome the AcMNPV complement sensitivity. One approach uses fusion proteins that provide protection against complement inactivation by displaying them on the $A c \mathrm{MNPV}$ viral envelope [35-37]. In the $B m \mathrm{NPV}$ vector, the surface structure of baculovirus BV (budded virus) may be a key factor responsible for the increased resistance to complement inactivation. $B m \mathrm{NPV}$ might serve as an alternative strategy that uses baculovirus vector in gene therapy applications.

Our EGFP and luciferase reporter gene delivery data in mice and chicks confirm that recombinant $B m \mathrm{NPV}$ exhibits complement resistance and can deliver foreign genes in vivo. In mice, luciferase expression was detected in many important organs: lung, liver, kidney, spleen, and brain. EGFP was also detected in these organs, except in liver, which exhibited fluorescence background too high to allow detection of EGFP fluorescence. To overcome this limitation, we also used a luciferase reporter gene. In chicks, the expression of the reporter genes was similar to mice. Additional expression was observed in the bursa of Fabricius. The luminescence and immunoblotting data showed that the luciferase expression was different in different tissues, indicating that the gene delivery effectiveness of $B m$ NPV differs among different tissues. The underlying mechanisms may involve a certain degree of complement inactivation, differences in baculovirus distribution, and its different half-lives in different tissues. Complement inactivation is still a key factor determining the success of baculovirus-based gene delivery [27]. In different tissues, exposure times of baculovirus to complement are different. Since $B m$ NPV vectors, like other gene delivery vectors, are exogenous and cannot replicate in vivo, they are cleared-up in tissues [38]. Thus, different organs and tissues differ in the distribution of baculovirus and other foreign genes.

Our luminescence results indicate that the luciferase expression efficiency in chicks is higher than in mice. The luciferase activity was detected in the heart, intestines, and testes of chicks, but it was not detected in those tissues in mice. The duration of recombinant $B m \mathrm{NPV}$-driven reporter gene expression in animals was shorter than that of recombinant $A c \mathrm{MNPV}$-driven expression in cells [30]. In addition, 17 days after virus infection, reporter gene expression disappeared because the baculovirus cannot replicate in animal cells [14], and its genomic DNA is less stable in tissues compared with cells. This feature ensures biosafety and makes the virus harmless to healthy animals and humans.

In IG and IV groups, EGFP and luciferase were detected in both mice and chicks. Our luminescence and Western blotting results showed that the tissue reporter gene expression was higher in the IG groups compared to the IV groups. However, in the IM groups, expression of EGFP and luciferase reporter genes was not detected. According to a previously published study [39], baculovirus-mediated gene delivery might be less efficient when administered via muscle. Other mechanisms may be involved in addition to the complement inactivation effect. By different administration routes, gene delivery vectors may enter the systemic circulation and be distributed to tissues through different pathways and with different absorption efficiencies [40].

The baculovirus system can be used for expression in vitro and ex vivo, and may also be effective for gene therapy in vivo. Early attempts were made to use the $A c \mathrm{MNPV}$ vector for transduction in mice. However, our results demonstrate that the $B m \mathrm{NPV}$ vector exhibits better characteristics for gene delivery in vertebrates. Additional modifications, such as surface shielding with decay acceleration factor, may help improve the efficiency of the recombinant $B m \mathrm{NPV}$ vector. Furthermore, the copy numbers of recombinant $B m \mathrm{NPV}$ viral genomes produced in one silkworm could reach $10^{12} \mathrm{vg}$, which provides higher efficiency for the production of recombinant baculovirus for gene delivery. The virtual inactivity of baculoviral promoters in vertebrate cells and their nonpathogenic nature in vertebrates ensure their safety for use in gene delivery. Our results indicate that the $B m \mathrm{NPV}$ vector exhibits a strong potential for gene therapy research and clinical applications.

\section{MATERIALS AND METHODS}

The study was conducted in accordance with animal ethics guidelines. All animal procedures were approved by the Beijing Administration Office of Laboratory Animals. The Bombyx mori-derived cell line Bm5 was cultured in TC100 insect cell culture medium (Applichem, Darmstadt, Germany) with 10\% fetal bovine serum (Gibco, USA) at $27^{\circ} \mathrm{C}$. VERO, PK-15, and Hela cells were cultured in Dulbecco's Modified Eagle Medium (DMEM, Gibco, USA) with $10 \%$ fetal bovine serum (FBS) at $37^{\circ} \mathrm{C}$. HEK293T cells were cultured in Minimum Eagle medium (MEM, Gibco, USA) with $10 \%$ FBS at $37^{\circ} \mathrm{C}$. E. coli Top10 cells were obtained from Invitrogen (Carlsbad, USA), and competent cells were prepared as described [41]. Eight-week-old C57BL/6 mice and 15-day-old specific pathogen-free (SPF) chicks were obtained from Vitalriver (Beijing, China). Human serum was obtained from Sigma-Aldrich (Deisenhofen, Germany). The reBmBac vector was constructed and prepared in our lab [42]. Expression of EGFP was analyzed by western blotting using the rabbit polyclonal anti-EGFP antibody (ab6556, Abcam, UK).

\section{Preparation of gene delivery viruses}

The CMV promoter was PCR-amplified (CMV-F: GGATATCTAGTTATTAATAGTAATCAATTACGG; 
CMV-R: CGGATCCGGATCTGACGGTTCACTAA ACCAGC) from the pEGFP-N3 vector (Invitrogen, Carlsbad, USA) and inserted into the pVL1393 transfer vector (Invitrogen) by EcoRV-BamHI digestion. Meanwhile the polyhedron promoter in pVL1393 was replaced. The SV40 polyadenylation signal was then PCR-amplified (SV40-F: TGCGGCCGCACTGCAGTC ATAATCAGCCATACCACATTTGT; SV40-R: GAGAT CTACATTGATGAGTTTGGACAAACC) from the pEGFP-N3 vector and inserted into the edited pVL1393 vector downstream of the CMV promoter by NotI-BglII digestion. The CMV promoter and SV40 polyadenylation signals were added to the transfer vector, which was named pVLCMV. The luciferase reporter gene was PCR-amplified (Luc-F: CGGATCCATGGAAGACGCCAAAAAC; Luc-R: GGA ATTCTTACACGGCGATCTTTCCGC) from the pGL3 vector (Promega, USA) and subcloned into the pVLCMV vector by EcoRI-BamHI digestion. This reaction produced the luciferase reporter gene transfer plasmid, which was named pVLCMV-luc. Similarly, the EGFP reporter gene transfer plasmid, pVLCMV-EGFP, was prepared by cloning the EGFP gene (EGFP-F: CGGATCCATGGTGAGCAAGGGCGAGGAG; EGFP-R: GGAATTCTTACTTGTACAGCTCGTCCATG) from pEGFP-N3 vector (Clontech, USA).

The recombinant $B m$ NPVs for reporter gene delivery, reBm-luc and reBm-EGFP, were prepared by cotransfection in Bm5 cells with reBmBac and pVLCMVluc or pVLCMV-EGFP as described $[42,43]$, and purified by plaque screening [44]. Fifth instar silkworm larvae were used to amplify the viruses; they were injected with recombinant $B m$ NPVs (approximately $10^{5}$ plaque forming units, p.f.u.). The recombinant $A c \mathrm{MNPV}$ reAc-luc, which was used to deliver the luciferase gene, was constructed in Sf9 cells by cotransfection of BacPAK6 (Clontech) and pVLCMV-luc as described [45]. The acquired reAcluc was then purified via a round of plaque purification [44]. Sf9 cells were infected with the purified virus at 0.1 MOI (multiplicity of infection of 0.1 p.f.u. per cell). Four days after infection, the larval hemolymph and cell-culture fluid were collected. The viruses were then purified and concentrated by centrifugation at 100,000 $\mathrm{g}$ for $80 \mathrm{~min}$ at $4^{\circ} \mathrm{C}$ through a $30 \%(\mathrm{w} / \mathrm{v})$ sucrose cushion [27].

\section{Quantitative polymerase chain reaction (Q-PCR) analysis of the viral genome}

Using the recombinant viruses as templates and primers (viral genome primers, VG-QF: GACA CCGAAACTCCGTATTGCC, VG-QR: ATCCGTTGA TTCCGTTGACACC; luc-QF: GGTGGACATCACTTA CGC, luc-QR: AATGCCCATACTGTTGAG; EGFPQF: CACAAGTTCAGCGTGTCCG, EGFP-QR: CTCGATGCGGTTCACCAG), Q-PCR was performed using the Toyobo SYBR Green. Standard curves were constructed based on serial dilutions of PCR products that ranged from $10^{12}$ to $10^{6}$ copies per microliter. The purified recombinant baculovirus solutions were quantified based on the Q-PCR results as the amount of virus genome per milliliter $(\mathrm{vg} / \mathrm{mL})$ [46]. The quantified results of the luciferase and EGFP reporter genes were compared with the viral genome results.

\section{Complement inactivation assay of baculoviruses}

VERO cells were cultured in 6-well plates at a constant cell density of $1 \times 10^{6}$ cells per well. Human serum was pretreated as described [27]. Purified reBm-luc or reAc-luc vectors with an MOI of 100 in the infection assay were incubated with different concentrations of human serum at $37^{\circ} \mathrm{C}$ for $30 \mathrm{~min}$, and then added to VERO cells for infection. Luciferase activity was measured $42 \mathrm{~h}$ later. The survival of the baculovirus vector was measured as the percentage of luciferase activity, which was compared with human serum heat-treated at $56^{\circ} \mathrm{C}$ as a control [27].

\section{Delivery of reporter genes in vitro}

VERO, PK-15, HEK293T, and Hela cells were cultured in 6-well plates at a constant cell density of $1 \times 10^{6}$ cells per well. Purified reBm-EGFP with an MOI of 100 was added into cultured cells for transduction. The treated cells were observed under an inverted fluorescence microscope (Nikon, Japan) $48 \mathrm{~h}$ later [12].

\section{Delivery of reporter genes in vivo}

The recombinant BmNPVs, reBm-EGFP and reBm-Luc vectors were used for gene delivery. Wildtype $B m$ NPV containing no reporter gene was used as a control. Intravenous injection group (IV): C57BL/6 mice were infected with control $B m \mathrm{NPV}$ or recombinant $B m \mathrm{NPV}$ s containing recombinant reporter genes via tail vein injection at $1 \times 10^{12}$ viral genome per kilogram $(\mathrm{vg} / \mathrm{kg})$; chicks were infected via wing vein injection at $1 \times 10^{12} \mathrm{vg} /$ $\mathrm{kg}$. Intramuscular injection group (IM): C57BL/6 mice and chicks were infected with control or delivery $B m$ NPVs via injection in the muscle of the quadriceps femoris or hind limb at $1 \times 10^{12} \mathrm{vg} / \mathrm{kg}$. Intragastric administration group (IG): C57BL/6 mice and chicks were starved for $12 \mathrm{~h}$ before gavage infusion with control or delivery $\mathrm{BmNPVs}$ at $1 \times 10^{13} \mathrm{vg} / \mathrm{kg}$. Three mice and three chicks were sacrificed by bloodletting $5,11,17,21$ and $30 \mathrm{~d}$ after recombinant baculovirus administration. Organs were excised for reporter gene assays. The EGFP-transfected organs were sectioned on a freezing microtome (CM3050S, Leica, Germany) or imaged by fluorescence microscope (Nikon, Japan). Homogenates (50 $\mu \mathrm{g}$ of protein) prepared from the reBmluc treated organs were assayed using a Luciferase Assay kit (Promega). The amount of protein in the homogenates was measured using the Bradford method [47]. 


\section{Author contributions}

X.L. and Z.Z. designed the experiments; Z.Z. supervised the project; X.L. performed experimental work and wrote the manuscript; Y.L. and X.H. performed experiment guidance; Y.Y. performed the expression experiments in silkworm. All authors reviewed the manuscript.

\section{CONFLICTS OF INTEREST}

The authors declare no conflicts of interest.

\section{FUNDING}

This work was supported by the National Natural Sciences Foundation of China (No. 31670156), the National Key Research and Development Program of China (No. 2016YFD0500108, 2017YFD0500706) and The State Key Laboratory of Integrated Management of Pest Insects and Rodents (Grant No. ChineseIPM1504).

\section{REFERENCES}

1. Lederberg J. (1963). Biological future of man. Ciba Foundation Symposium-Man and His Future. (Wiley Online Library), pp. 263-273.

2. Friedmann T, Roblin R. Gene therapy for human genetic disease? Science. 1972; 175: 949-955.

3. Wigler M, Silverstein S, Lee LS, Pellicer A, Cheng Y, Axel R. Transfer of purified herpes virus thymidine kinase gene to cultured mouse cells. Cell. 1977; 11: 223-232.

4. Blaese RM, Culver KW, Miller AD, Carter CS, Fleisher T, Clerici M, Shearer G, Chang L, Chiang Y, Tolstoshev P, Greenblatt JJ, Rosenberg SA, Klein H, et al. T lymphocytedirected gene therapy for ADA- SCID: initial trial results after 4 years. Science. 1995; 270: 475-480.

5. Kaplan JM. Adenovirus-based cancer gene therapy. Curr Gene Ther. 2005; 5: 595-605.

6. Ginn SL, Alexander IE, Edelstein ML, Abedi MR, Wixon J. Gene therapy clinical trials worldwide to 2012 - an update. J Gene Med. 2013; 15: 65-77.

7. Solinis MA, Del Pozo-Rodriguez A, Apaolaza PS, Rodriguez-Gascon A. Treatment of ocular disorders by gene therapy. Eur J Pharm Biopharm. 2014.

8. Giacca M. (2010). Methods for Gene Delivery. Gene Therapy. (Milano: Springer Milan), pp. 47-137.

9. Hofmann C, Sandig V, Jennings G, Rudolph M, Schlag P, Strauss M. Efficient gene transfer into human hepatocytes by baculovirus vectors. Proc Natl Acad Sci U S A. 1995; 92: 10099-10103.

10. Boyce FM, Bucher NL. Baculovirus-mediated gene transfer into mammalian cells. Proc Natl Acad Sci U S A. 1996; 93: 2348-2352.
11. Kost T, Condreay J. Innovations-biotechnology: baculovirus vectors as gene transfer vectors for mammalian cells: biosafety considerations. Appl Biosaf. 2002; 7: 167-169.

12. Gao H, Wang Y, Li N, Peng WP, Sun Y, Tong GZ, Qiu HJ. Efficient gene delivery into mammalian cells mediated by a recombinant baculovirus containing a whispovirus ie 1 promoter, a novel shuttle promoter between insect cells and mammalian cells. J Biotechnol. 2007; 131: 138-143.

13. Brusca J, Summers M, Couch J, Courtney L. Autographa californica nuclear polyhedrosis virus efficiently enters but does not replicate in poikilothermic vertebrate cells. Intervirology. 1986; 26: 207-222.

14. Hartig PC, Cardon MC, Kawanishi CY. Insect virus: assays for viral replication and persistence in mammalian cells. $\mathrm{J}$ Virol Methods. 1991; 31: 335-344.

15. Chen CY, Lin CY, Chen GY, Hu YC. Baculovirus as a gene delivery vector: recent understandings of molecular alterations in transduced cells and latest applications. Biotechnol Adv. 2011; 29: 618-631.

16. Wu YL, Wu CP, Huang YH, Huang SP, Lo HR, Chang HS, Lin $\mathrm{PH}, \mathrm{Wu} \mathrm{MC}$, Chang CJ, Chao YC. Identification of a high-efficiency baculovirus DNA replication origin that functions in insect and mammalian cells. J Virol. 2014; 88: 13073-13085.

17. Hu YC. Baculovirus vectors for gene therapy. Adv Virus Res. 2006; 68: 287-320.

18. Sarkis C, Serguera C, Petres S, Buchet D, Ridet JL, Edelman L, Mallet J. Efficient transduction of neural cells in vitro and in vivo by a baculovirus-derived vector. Proc Natl Acad Sci U S A. 2000; 97: 14638-14643.

19. Kaikkonen MU, Raty JK, Airenne KJ, Wirth T, Heikura $\mathrm{T}$, Yla-Herttuala $\mathrm{S}$. Truncated vesicular stomatitis virus $\mathrm{G}$ protein improves baculovirus transduction efficiency in vitro and in vivo. Gene Ther. 2006; 13: 304-312.

20. Park HJ, Lee WY, Kim JH, Kim JH, Jung HJ, Kim NH, Kim $\mathrm{BK}$, Song H. Interstitial tissue-specific gene expression in mouse testis by intra-tunica albuguineal injection of recombinant baculovirus. Asian J Androl. 2009; 11: 342-350.

21. Airenne KJ, Makkonen KE, Mahonen AJ, Yla-Herttuala S. In vivo application and tracking of baculovirus. Curr Gene Ther. 2010; 10: 187-194.

22. Kinnunen K, Kalesnykas G, Mahonen AJ, Laidinen S, Holma L, Heikura T, Airenne K, Uusitalo H, Yla-Herttuala S. Baculovirus is an efficient vector for the transduction of the eye: comparison of baculovirus- and adenovirus-mediated intravitreal vascular endothelial growth factor $\mathrm{D}$ gene transfer in the rabbit eye. J Gene Med. 2009; 11: 382-389.

23. Turunen TA, Laakkonen JP, Alasaarela L, Airenne KJ, YlaHerttuala S. Sleeping Beauty-baculovirus hybrid vectors for long-term gene expression in the eye. J Gene Med. 2014; 16: 40-53.

24. Wang S, Balasundaram G. Potential cancer gene therapy by baculoviral transduction. Curr Gene Ther. 2010; 10: 214-225. 
25. Luo WY, Shih YS, Lo WH, Chen HR, Wang SC, Wang CH, Chien $\mathrm{CH}$, Chiang CS, Chuang YJ, Hu YC. Baculovirus vectors for antiangiogenesis-based cancer gene therapy. Cancer Gene Ther. 2011; 18: 637-645.

26. Luo WY, Shih YS, Hung CL, Lo KW, Chiang CS, Lo WH, Huang SF, Wang SC, Yu CF, Chien CH, Hu YC. Development of the hybrid Sleeping Beauty-baculovirus vector for sustained gene expression and cancer therapy. Gene Ther. 2012; 19: 844-851.

27. Hofmann C, Strauss M. Baculovirus-mediated gene transfer in the presence of human serum or blood facilitated by inhibition of the complement system. Gene Ther. 1998; 5: 531-536.

28. Maeda S, Kamita SG, Kondo A. Host range expansion of Autographa californica nuclear polyhedrosis virus (NPV) following recombination of a 0.6-kilobase-pair DNA fragment originating from Bombyx mori NPV. J Virol. 1993; 67: 6234-6238.

29. van Oers MM, Pijlman GP, Vlak JM. Thirty years of baculovirus-insect cell protein expression: from dark horse to mainstream technology. J Gen Virol. 2015; 96: 6-23.

30. Kenoutis C, Efrose RC, Swevers L, Lavdas AA, Gaitanou M, Matsas R, Iatrou K. Baculovirus-mediated gene delivery into mammalian cells does not alter their transcriptional and differentiating potential but is accompanied by early viral gene expression. J Virol. 2006; 80: 4135-4146.

31. Spenger A, Ernst W, Condreay JP, Kost TA, Grabherr R. Influence of promoter choice and trichostatin A treatment on expression of baculovirus delivered genes in mammalian cells. Protein Expr Purif. 2004; 38: 17-23.

32. Chen HC, Lee HP, Ho YC, Sung ML, Hu YC. Combination of baculovirus-mediated gene transfer and rotating-shaft bioreactor for cartilage tissue engineering. Biomaterials. 2006; 27: 3154-3162.

33. Smith RH, Levy JR, Kotin RM. A simplified baculovirusAAV expression vector system coupled with one-step affinity purification yields high-titer rAAV stocks from insect cells. Mol Ther. 2009; 17: 1888-1896.

34. Galibert L, Merten OW. Latest developments in the largescale production of adeno-associated virus vectors in insect cells toward the treatment of neuromuscular diseases. J Invertebr Pathol. 2011; 107: S80-93.

35. Spitzer D, Hauser H, Wirth D. Complement-protected amphotropic retroviruses from murine packaging cells. Hum Gene Ther. 1999; 10: 1893-1902.
36. Huser A, Rudolph M, Hofmann C. Incorporation of decayaccelerating factor into the baculovirus envelope generates complement-resistant gene transfer vectors. Nat Biotechnol. 2001; 19: 451-455.

37. Guibinga GH, Friedmann T. Baculovirus GP64pseudotyped HIV-based lentivirus vectors are stabilized against complement inactivation by codisplay of decay accelerating factor (DAF) or of a GP64-DAF fusion protein. Mol Ther. 2005; 11: 645-651.

38. Kotchey NM, Adachi K, Zahid M, Inagaki K, Charan R, Parker RS, Nakai H. A potential role of distinctively delayed blood clearance of recombinant adeno-associated virus serotype 9 in robust cardiac transduction. Mol Ther. 2011; 19: 1079-1089.

39. Pieroni L, Maione D, La Monica N. In vivo gene transfer in mouse skeletal muscle mediated by baculovirus vectors. Hum Gene Ther. 2001; 12: 871-881.

40. Masuda I, Matsuo T, Yasuda T, Matsuo N. Gene transfer with liposomes to the intraocular tissues by different routes of administration. Invest Ophthalmol Vis Sci. 1996; 37: 1914-1920.

41. Sambrook J, Russell DW. The Hanahan method for preparation and transformation of competent E. coli: highefficiency transformation. CSH Protoc. 2006.

42. Liu X, Wei Y, Li Y, Li H, Yang X, Yi Y, Zhang Z. A Highly efficient and simple construction strategy for producing recombinant baculovirus Bombyx mori nucleopolyhedrovirus. PLoS One. 2016; 11: e0152140.

43. Groebe DR, Chung AE, Ho C. Cationic lipid-mediated co-transfection of insect cells. Nucleic Acids Res. 1990; 18: 4033.

44. Pen J, Welling GW, Welling-Wester S. An efficient procedure for the isolation of recombinant baculovirus. Nucleic Acids Res. 1989; 17: 451.

45. Kitts PA, Possee RD. A method for producing recombinant baculovirus expression vectors at high frequency. Biotechniques. 1993; 14: 810-817.

46. Hitchman RB, Siaterli EA, Nixon CP, King LA. Quantitative real-time PCR for rapid and accurate titration of recombinant baculovirus particles. Biotechnol Bioeng. 2007; 96: 810-814.

47. Kruger NJ. The Bradford method for protein quantitation. Methods Mol Biol. 1994; 32: 9-15. 